\title{
Many-Body Quantum Chaos: Analytic Connection to Random Matrix Theory
}

\author{
Pavel Kos, Marko Ljubotina, and Tomaž Prosen* \\ Physics Department, Faculty of Mathematics and Physics, University of Ljubljana, \\ Jadranska 19, SI-1000 Ljubljana, Slovenia
}

(Received 5 February 2018; revised manuscript received 12 April 2018; published 8 June 2018)

\begin{abstract}
A key goal of quantum chaos is to establish a relationship between widely observed universal spectral fluctuations of clean quantum systems and random matrix theory (RMT). Most prominent features of such RMT behavior with respect to a random spectrum, both encompassed in the spectral pair correlation function, are statistical suppression of small level spacings (correlation hole) and enhanced stiffness of the spectrum at large spectral ranges. For single-particle systems with fully chaotic classical counterparts, the problem has been partly solved by Berry [Proc. R. Soc. A 400, 229 (1985)] within the so-called diagonal approximation of semiclassical periodic-orbit sums, while the derivation of the full RMT spectral form factor $K(t)$ (Fourier transform of the spectral pair correlation function) from semiclassics has been completed by Müller et al. [Phys. Rev. Lett. 93, 014103 (2004)]. In recent years, the questions of long-time dynamics at high energies, for which the full many-body energy spectrum becomes relevant, are coming to the forefront even for simple many-body quantum systems, such as locally interacting spin chains. Such systems display two universal types of behaviour which are termed the "many-body localized phase" and "ergodic phase." In the ergodic phase, the spectral fluctuations are excellently described by RMT, even for very simple interactions and in the absence of any external source of disorder. Here we provide a clear theoretical explanation for these observations. We compute $K(t)$ in the leading two orders in $t$ and show its agreement with RMT for nonintegrable, time-reversal invariant many-body systems without classical counterparts, a generic example of which are Ising spin-1/2 models in a periodically kicking transverse field. In particular, we relate $K(t)$ to partition functions of a class of twisted classical Ising models on a ring of size $t$; hence, the leading-order RMT behavior $K(t) \simeq 2 t$ is a consequence of translation and reflection symmetry of the Ising partition function.
\end{abstract}

DOI: 10.1103/PhysRevX.8.021062

Subject Areas: Quantum Physics, Statistical Physics, Strongly Correlated Materials

\section{INTRODUCTION}

Random matrix theory (RMT) was introduced into physics in the 1950s by Wigner [1] to provide a statistical description of nuclear resonance or excitation spectra. It should be intuitively clear that a system consisting of a few tens of nucleons coupled via short- and long-range interactions is complicated enough that a successful description of experimental spectral fluctuations in terms of an ensemble of random Hamiltonians with independent stochastic matrix elements is not that surprising. An example of a robust phenomenological measure of fluctuations is the statistical variance of the number of energy levels in an

\footnotetext{
* Corresponding author. tomaz.prosen@fmf.uni-lj.si

Published by the American Physical Society under the terms of the Creative Commons Attribution 4.0 International license. Further distribution of this work must maintain attribution to the author(s) and the published article's title, journal citation, and DOI.
}

interval of fixed length $\Delta E$ which, in RMT and experimental nuclear spectra [2], grows as $\sim \log |\bar{\rho} \Delta E|$ (known as spectral stiffness), rather than $\sim \sqrt{\bar{\rho} \Delta E}$ as in the Poissonian random spectrum ( $\bar{\rho}$ is the average density of states). The atomic spectra observed already by 1960 exhibited the so-called "level repulsion," which can be quantitatively explained [3] with Wigner's RMT. However, in the early 1980s a much more surprising fact was revealed, namely, that RMT also works extremely well for capturing spectral fluctuations of simple single-particle systems whose corresponding classical dynamics are completely chaotic, such as dispersive (Sinai) billiards or hydrogen or Rydberg atoms in external magnetic or microwave fields. These observations [4-6], termed the quantum chaos conjecture, which has been concisely stated in Ref. [7], have driven the field of quantum chaos for decades. The first, partial explanation for the success of RMT in simple chaotic systems came from Berry's semiclassical (small effective $\hbar$ ) calculation [8] of the spectral form factor $K(t)$ in terms of a double sum over classical unstable periodic orbits, which we explain below. $K(t)$ is 
defined as a Fourier transformation of the two-point correlation function of the spectral density $\rho(E)=\sum_{j} \delta\left(E-E_{j}\right)$, with $\left\{E_{j}\right\}$ being the energy spectrum,

$$
K(t)=\int e^{-i \epsilon t / \hbar}\left[\left\langle\rho\left(E+\frac{\epsilon}{2}\right) \rho\left(E-\frac{\epsilon}{2}\right)\right\rangle_{E}-\bar{\rho}^{2}\right] \frac{d \epsilon}{\bar{\rho}},
$$

where $\bar{\rho}=\langle\rho(E)\rangle_{E}$ and $\langle\cdots\rangle_{E}$ represents local energy average over an energy shell (say, of width $\Delta E$ ) containing many levels $\bar{\rho} \Delta E \gg 1$, in the case of autonomous (timeindependent) systems. In the case of periodically driven, i.e., Floquet systems, that we discuss later in this paper, the average over the full range $[0,2 \pi)$ of quasienergieseigenvalues of the unitary Floquet (one-period) propagator $U$-is normally considered as the physical properties are not expected to depend on the particular value of quasienergy. A fruitful intuition stems from the observation that $K(t)$ characterizes all pair-correlation properties including the level repulsion and spectral stiffness, since in RMT $\left\{E_{j}\right\}$ can be considered as a fictitious one-dimensional (Dyson's) gas with a logarithmic pairwise interaction [9].

For integrable systems, possessing a complete set of conserved quantities, the energy spectrum $\left\{E_{j}\right\}$ is conjectured [10] to represent a Poisson random uncorrelated sequence, so the spectral form factor Eq. (1) can be exactly computed as $K(t) \equiv t_{H}=2 \pi \hbar \bar{\rho}=$ const for all $t>0$, and thus provides a clear discriminator between integrable and chaotic systems, since for the latter $K(t) \propto t$, in agreement with explicit predictions of RMT, as we explain in Sec. I A.

A clear heuristic derivation of the RMT spectral form factor $K(t)$ for classically strongly chaotic (hyperbolic) systems from semiclassical periodic orbit theory, starting from Berry's diagonal approximation [8], upgraded to second order in $t$ by Sieber and Richter [11,12], and finally completed to all orders in a tour de force by Müller et al. $[13,14]$, has been arguably the main accomplishment of the field of quantum chaos of single- or few-particle systems. Nevertheless, a rigorous proof of the quantum chaos conjecture has so far only been possible for a much more abstract class of single-particle systems, specifically for mixing quantum graphs $[15,16]$. These semiclassical periodic-orbit approaches have a natural generalization to a quantum many-body problem for bosons when the number of quanta per mode is large [17-20].

However, RMT has also been found to excellently describe spectral fluctuations in the simplest, say, lowdimensional and locally interacting, nonintegrable manybody systems where local degrees of freedom have no classical limit at all, such as spin-1/2's, qubits, fermions, etc. [21-24], and where no semiclassical or mean-field approach can be applied. Because of such phenomenological success, RMT statistics of level spacings is nowadays used essentially as a definition of the so-called quantum chaotic or ergodic phase (see, e.g., Refs. [25-30]). Moreover, the ergodic phase has been intensively theoretically investigated in recent years and its most concise characterization is provided by the so-called eigenstate thermalization hypothesis (see, e.g., Refs. [25,31]). Nevertheless, there has so far been no proposition of the underlying dynamical (or microscopic) mechanism (such as unstable periodic orbit pairings in semiclassical chaotic systems discussed above). Recent studies of out-of-time-ordered correlations in many-body systems, some of which establish exponential growth [in particular in $(0+1)$-dimensional systems such as the Sachdev-Ye-Kitaev model], have no clear connection to Lyapunov instability, as it is understood in classical dynamical systems theory, and is the only mathematically meaningful definition of chaos. This has to do with the lack of the concept of classical orbits and the corresponding unstable (nonlinear) equations of motion, which result in sensitive dependence on initial conditions (e.g., the butterfly effect). In short, the concept of orbits and Lyapunov chaos does not make sense at $\hbar \sim 1$. One thus urgently needs alternative concepts which would enable one to explain the surprising success of RMT in simple many-qubit systems.

Providing one such concept is the main objective of this article. We identify a coherent structure, in a class of generic many-body quantum systems with the lowest, twodimensional local Hilbert space (qubits, or spin-1/2's), which is responsible for building up level (spectral) correlations. Expanding $K(t)$, which is written as the product of two traces of the quantum mechanical propagator (see Sec. IA), in the computational spin basis and writing it in a discrete-path-integral-like fashion, we find that the leading contribution comes from constructive interference, which corresponds to a partition function of a classical one-dimensional Ising model. Furthermore, subleading contributions can be interpreted as a family of partition functions of so-called twisted Ising models which are classified using a novel diagrammatic technique. In terms of this expansion, the leading contributions to $K(t)$ are shown to exactly correspond to RMT results $K(t) \simeq 2 t$ for times longer than a certain crossover time $t^{*}$, while the nonuniversal results at $t<t^{*}$ are shown to reproduce numerical data extremely well. The timescale $t^{*}$, which scales logarithmically with the system size, can be interpreted as a quantum many-body analogue of the Ehrenfest (or Thouless) time. Finally, we identify the nonsemiclassical analog of the Sieber-Richter pairing mechanism [11] and exactly reproduce the subleading RMT term $-2 t^{2} / t_{H}$ as well.

\section{A. Spectral form factor in Floquet systems and periodic orbit theory}

In order to address the setup with a minimal amount of inessential technical complications, we decided to study periodically driven (Floquet) many-body systems in the 
absence of any conserved charges or unitary symmetries. Even in a single-particle context, these are the minimal models of quantum chaos [9] and correspond to Dyson's circular ensembles of random unitary matrices [32].

In this section we define the main object of our study, namely, the spectral form factor for Floquet systems, and for comparison with the main derivation in Sec. II of our paper, we outline the key steps of historical semiclassical derivation of the RMT form factor in terms of periodic-orbit theory. For a unitary one-period Floquet propagator $U$, we write the eigenphases $\varphi_{n}$ and eigenvectors $|n\rangle$ as $U|n\rangle=e^{-i \varphi_{n}}|n\rangle, n=1, \ldots, \mathcal{N}$, where $\mathcal{N}$ denotes the dimension of the Hilbert space. The spectral density (one-point function) is now defined as

$$
\rho(\varphi)=\frac{2 \pi}{\mathcal{N}} \sum_{n} \delta\left(\varphi-\varphi_{n}\right)
$$

and is normalized to a unit mean level density

$$
\langle\rho(\varphi)\rangle_{\varphi} \equiv \frac{1}{2 \pi} \int_{0}^{2 \pi} d \varphi \rho(\varphi)=1 .
$$

Locally averaged density is expected to be $\varphi$ independent, which makes Floquet systems particularly appealing for studying spectral fluctuations. These are encapsulated in the connected (two-point) spectral correlation function,

$$
R(\vartheta)=\langle\rho(\varphi+\vartheta / 2) \rho(\varphi-\vartheta / 2)\rangle_{\varphi}-\langle\rho\rangle_{\varphi}^{2},
$$

which is, again, expected to be homogeneous ( $\varphi$ independent). An equivalent, and very convenient quantity, is the spectral form factor $K(t), t \in \mathbb{Z}$, defined as an appropriately scaled Fourier transform:

$$
\begin{aligned}
K(t) & =\frac{\mathcal{N}^{2}}{2 \pi} \int_{0}^{2 \pi} d \vartheta R(\vartheta) e^{-i \vartheta t} \\
& =\sum_{n} e^{-i t \varphi_{n}} \sum_{n^{\prime}} e^{i t \varphi_{n^{\prime}}}-\mathcal{N}^{2} \delta_{t, 0}=\left|\operatorname{tr} U^{t}\right|^{2}-\mathcal{N}^{2} \delta_{t, 0} .
\end{aligned}
$$

Finally, one writes

$$
K(t)=\left\langle\left(\operatorname{tr} U^{t}\right)\left(\operatorname{tr} U^{-t}\right)\right\rangle-\mathcal{N}^{2} \delta_{t, 0},
$$

where $\langle\cdots\rangle$ represents an appropriate additional averaging, either over local windows of time $t$ (moving time average) or over an ensemble of similar systems, which is needed since the spectral form factor Eq. (5) is not a self-averaging quantity [33].

For circular random matrix ensembles [32], which are expected to model Floquet systems in RMT [orthogonal (OE) ensemble or unitary ensemble (UE) for systems with or without time-reversal or more general antiunitary symmetry], the spectral form factor up to Heisenberg time, $t<\mathcal{N}$, reads

$$
\begin{gathered}
K_{\mathrm{OE}}(t)=2 t-t \ln (1+2 t / \mathcal{N})=2 t-2 t^{2} / \mathcal{N}+\cdots, \\
K_{\mathrm{UE}}(t)=t .
\end{gathered}
$$

Note that exactly the same expressions hold as well for Gaussian ensembles of RMT which model timeindependent systems.

For Floquet systems with a well-defined classical limit, where the motion is hyperbolic (chaotic) everywhere in the phase space, one can write tr $U^{t}$ in terms of a Feynman path integral and evaluate it by the method of stationary phase in terms of a finite sum over all periodic orbits $p$ of length $t$, with classical actions $S_{p}$, and amplitudes $A_{p}$ which are proportional to the inverse square root of stability exponents:

$$
\operatorname{tr} U^{t} \simeq \sum_{p} A_{p} e^{-i S_{p} / \hbar}
$$

Note that we choose to work at fixed $t$ rather than at fixed energy $E$ as is customary in semiclassical analysis of timeindependent systems. Here one assumes that the effective $\hbar$ is small, i.e., $S_{p} \gg \hbar$ for all $p$, which is justified for large Hilbert space dimensions $\mathcal{N} \gg 1$. A semiclassical representation of the spectral form factor can then be written as

$$
K(t) \simeq\left\langle\sum_{p, p^{\prime}} A_{p} A_{p^{\prime}}^{*} e^{-i\left(S_{p}-S_{p^{\prime}}\right) / \hbar}\right\rangle .
$$

Berry identified the leading RMT contribution $K(t) \simeq t$ from the diagonal terms of paired orbits, arguing that the nondiagonal terms of unequal orbit pairs average out in the leading order due to random phases. The diagonal contribution then results from the Hannay-Ozorido de Almeida sum rule [34], $\sum_{p}\left|A_{p}\right|^{2}=t$, which is just a restatement of classical ergodicity. For systems with time-reversal invariance, the leading order of the RMT result Eq. (7), $K(t) \simeq 2 t$, then simply follows by pairing each orbit with itself $p^{\prime}=p$ and its time-reversed partner $p^{\prime}=\bar{p}$, noting that $S_{\bar{p}}=S_{p}$ and $A_{\bar{p}}=A_{p}$. However, Berry's result only holds on timescales much shorter than the Heisenberg time $t \ll t_{H}$, which translates to spectral correlations on quasienergy ranges much larger than the mean level spacing. That result can thus be considered as the leading order of a power-series expansion (in $t$ ) of the RMT expression for $K(t)$. Further progress came only 16 years later when Sieber and Richter [11,12] correctly identified the next-to-leading RMT term of $K(t)=2 t-$ $2 t^{2} / t_{H}+\mathcal{O}\left(t^{3} / t_{H}^{2}\right)$ for time-reversal invariant systems via the self-encountering periodic orbit doublets. Specifically, they decomposed the periodic-orbit sum into two parts, the first containing a majority of orbits which never come close to themselves before the full period, and in the second part, they considered orbits which experience a close self- 
encounter. They argued that the orbits from the second group form doublets with very similar actions $S_{p}$ and amplitudes $A_{p}$, which thus coherently interfere in the double sum Eq. (10) and result, after careful bookkeeping, exactly in the second-order term $-2 t^{2} / t_{H}$ of RMT. It took another few years of efforts until this endeavor was finally completed in Ref. [13,14] (see also Ref. [35] for the analysis of unitary-to-orthogonal ensemble crossover) by correctly identifying all the terms in the power-series expansion of $K(t)$ from sums over chaotic periodic orbits with an arbitrary number of self-encounters.

\section{PARTITION FUNCTION EXPANSION OF THE SPECTRAL FORM FACTOR}

Here, however, we consider an interacting many-body system of quantum excitations without any meaningful classical limit, so the semiclassical periodic orbit is not applicable. We consider a system of $\ell$ spin-1/2's (qubits) described by Pauli spin operators $\sigma_{x}^{(\alpha)}, \alpha \in\{1,2,3\}$, $x \in\{1, \ldots, \ell\}$, where the time evolution is given by the following two-step unitary Floquet propagator of a periodically pulse-driven Hamiltonian,

$$
H(t)=H_{0}+H_{1} \sum_{m \in \mathbb{Z}} \delta(t-m)
$$

(time is measured in units of pulse period),

$$
\begin{aligned}
& U=\mathcal{T}-\exp \left(-i \int_{0}^{1} d t H(t)\right)=V W, \\
& W=e^{-i H_{0}}, \quad H_{0}=\sum_{x} J_{x}^{1} \sigma_{x}^{(3)}+\sum_{x<x^{\prime}} J_{x, x^{\prime}}^{2} \sigma_{x}^{(3)} \sigma_{x^{\prime}}^{(3)}+\cdots, \\
& V=e^{-i H_{1}}=v^{\otimes \ell}, \quad H_{1}=h \sum_{x} \sigma_{x}^{(1)},
\end{aligned}
$$

where $v$ is a $2 \times 2$ matrix with elements $v_{00}=v_{11}=\cos h$, $v_{01}=v_{10}=-i \sin h$. In the basis of $\mathcal{N}=2^{\ell}$ joint eigenstates of $\sigma_{x}^{(3)}, \sigma_{x}^{(3)}|\underline{s}\rangle=(-1)^{s_{x}}|\underline{s}\rangle$, labeled by classical spin configurations $\underline{s}=\left(s_{1}, \ldots, s_{\ell}\right), s_{x} \in\{0,1\}, W$ acts as a pure phase factor,

$$
\begin{gathered}
W|\underline{s}\rangle=e^{-i \theta_{\underline{s}}}|\underline{s}\rangle, \\
\theta_{\underline{s}}=\sum_{x} J_{x}^{1}(-1)^{s_{x}}+\sum_{x<x^{\prime}} J_{x, x^{\prime}}^{2}(-1)^{s_{x}+s_{x^{\prime}}}+\cdots
\end{gathered}
$$

while the matrix elements of $V$ factorize

$$
\left\langle\underline{s}|V| \underline{s}^{\prime}\right\rangle=\prod_{x=1}^{\ell} v_{s_{x}, s_{x}^{\prime}} .
$$

The propagator Eq. (12) defines a generic family of Ising models periodically kicked with a uniform transverse field (generalizing the kicked Ising chain [36,37], where RMT spectral fluctuations have been verified to a high precision [38]). See also Ref. [39] for a related discussion of transfermatrix evaluation of the many-body propagator. In more abstract terms, one can also view $H_{0}$ as a generic integrable or many-body localized system with $l$-bits $\sigma_{x}^{(3)}$ and $V$ as a global perturbation. The model is time-reversal invariant as the matrices of $H_{0,1}$ are real; i.e., $V, W$ are symmetric.

We note immediately that the method that we develop below can be used as well to study a continuous-time version of the transverse field Ising model, where the kicked model Eq. (11) represents its trotterization (via the Trotter formula) by substituting $J_{x \ldots}^{k} \rightarrow(\Delta t) J_{x \ldots}^{k}$, $h \rightarrow(\Delta t) h$, and carefully performing double scaling $\ell \rightarrow$ $\infty$ and $\Delta t \rightarrow 0$ where the thermodynamic limit should be considered first. Further, more general forms of offdiagonal perturbations $V$ can be considered by allowing an arbitrary spatial dependence of the magnetic field $h \rightarrow h_{x}$. Nonetheless, the particular system that we choose to study in the present paper represents a minimal generic model of many-body quantum chaos at $\hbar \sim 1$.

We start by considering an expression Eq. (6) for the spectral form factor of Floquet systems $K(t)=$ $\left\langle\left(\operatorname{tr} U^{t}\right)\left(\operatorname{tr} U^{-t}\right)\right\rangle$, defined for positive integer time $t$. Inserting multiple identities $\sum_{\underline{s}_{\tau}}\left|\underline{s}_{\tau}\right\rangle\left\langle\underline{s}_{\tau}\right|=1$ in $\operatorname{tr} U^{t}$ and $\sum_{\underline{s}_{\tau}^{\prime}}\left|\underline{s}_{\tau}^{\prime}\right\rangle\left\langle\underline{s}_{\tau}^{\prime}\right|=1$ in $\operatorname{tr} U^{-t}$, we obtain

$$
\begin{aligned}
K(t)= & \sum_{\underline{s}_{1}, \ldots, \underline{s}_{t}} \sum_{s_{1}^{\prime}, \ldots, \underline{s}_{t}^{\prime}}\left\langle e^{-i \sum_{\tau=1}^{t}\left(\theta_{\underline{s}_{\tau}}-\theta_{\underline{s}_{\tau}^{\prime}}\right)}\right\rangle \\
& \times \prod_{x=1}^{\ell} \prod_{\tau=1}^{t} v_{s_{x, \tau}, s_{x, \tau+1}} v_{s_{x, \tau}^{\prime}, s_{x, \tau+1}^{\prime}}^{*} .
\end{aligned}
$$

Note that taking the trace implies periodic boundary conditions in time $t+1 \equiv 1$. Assuming pseudorandomness of the phases $\theta_{\underline{s}}$, one has

$\left\langle e^{-i \sum_{\tau=1}^{t}\left(\theta_{\underline{s}_{\tau}}-\theta_{\underline{S}_{\tau}^{\prime}}\right)}\right\rangle=\delta_{\left\langle\underline{s}_{1}, \ldots, \underline{s}_{t}\right\rangle,\left\langle\underline{\underline{s}}_{1}^{\prime}, \ldots, \underline{s}_{t}^{\prime}\right\rangle}+$ fluctuations,

where $\left\langle\underline{s}_{1}, \underline{s}_{2}, \ldots, \underline{s}_{t}\right\rangle$ represents a lexicographically ordered string of words $\underline{s}_{1}, \underline{s}_{2}, \ldots, \underline{s}_{t}$. In the ideal case, where all $2^{\ell}$ phases $\theta_{\underline{s}}$ can be assumed to be independent random and uniform in $[0,2 \pi)$ (which is equivalent to the assumption that all the coupling constants $J_{x, x^{\prime} \ldots}^{k}$ are independent identically distributed), the fluctuation term in Eq. (17) exactly vanishes.

We refer to such an ideal situation in which fluctuations in Eq. (17) are set to zero as a random phase model (RPM). Below we show how to compute $K(t)$ for the RPM and demonstrate that the result describes both the universal (RMT) and nonuniversal (short-time) regimes of large families of clean kicked Ising models excellently. 
For times much shorter than the Heisenberg time $t_{H}=2^{\ell}$, one may assume that all configurations $\underline{s}_{\tau}$ in the string $\underline{s}_{1}, \underline{s}_{2}, \ldots, \underline{s}_{t}$ are different. Then, Eq. (17) implies that there exists a permutation $\pi \in S_{t}: \tau \rightarrow \pi(\tau)$, such that $\underline{s}_{\tau}^{\prime}=\underline{s}_{\pi(\tau)} ;$ therefore,

$$
K(t)=\sum_{\pi \in S_{t}} Z_{\pi}^{\ell}
$$

where

$$
Z_{\pi}=\sum_{s_{1}, \ldots, s_{t}} \prod_{\tau=1}^{t} v_{s_{\tau}, s_{\tau+1}} v_{s_{\pi(\tau)}, s_{\pi(\tau+1)}}^{*},
$$

up to $\mathcal{O}\left(t / 2^{\ell}\right)$. Denoting by $w(\underline{s})=\frac{1}{2} \sum_{\tau=1}^{t}\left(1-\delta_{s_{\tau}, s_{\tau+1}}\right)$ a half-number of domain walls in a periodic spin sequence $\underline{s}$ (which is always an integer), $Z_{\pi}$ can be rewritten as

$$
Z_{\pi}=(\cos h)^{2 t} \sum_{\underline{s} \in\{0,1\}^{t}}(-|\tan h|)^{w(\underline{s})+w(\pi(\underline{s}))} .
$$

Note that for the identity permutation, $Z_{\text {id }}$ is a partition function of a classical one-dimensional Ising model (on a ring of circumference $t$ ) which can be calculated via a $2 \times 2$ transfer matrix $T_{s s^{\prime}}=\left|v_{s s^{\prime}}\right|^{2}$, namely, $Z_{\text {id }}=\operatorname{tr} T^{t}=1+(\cos 2 h)^{t} . Z_{\pi}$ equals $Z_{\text {id }}$ for any other permutation which does not change any neighbors in the string $\underline{s}$, i.e., conserves the domain wall counting function $w(\underline{s})$. These are exactly the $t$ cyclic permutations and $t$ anticyclic permutations - compositions of cyclic permutations with inversion $\tau \rightarrow t+1-\tau$. For all other permutations $\pi$ which contain at least one pair of neighbor changes, one can show that the twisted partition functions $Z_{\pi \neq i d}$ are strictly smaller, and can be systematically computed using a diagrammatic technique (see Appendix A). Upon approaching the thermodynamic limit $\ell \rightarrow \infty$ at fixed $t$, one thus finds an exact asymptotic result:

$$
\begin{aligned}
K(t) & \simeq 2 t\left[1+(\cos 2 h)^{t}\right]^{\ell} \\
& \simeq 2 t \quad \text { for } t \gg t^{*} \\
t^{*} & =-\frac{\ln \ell}{\ln \cos 2 h} .
\end{aligned}
$$

This result can be interpreted as an analogue of Berry's diagonal approximation and yields the first order of RMT [Eq. (7)], while exact nonuniversal behavior is predicted for times $t \lesssim t^{*}=\mathcal{O}\left(h^{-2} \ln \ell\right)$. The timescale $t^{*}$ which separates the universal from nonuniversal behavior can be interpreted as a kind of quantum many-body Ehrenfest time. Note that in the limit $h \rightarrow 0$, the spectral form factor grows to the saturation value $\mathcal{N}$ at $t \sim 1$, and one obtains the expected Poissonian behavior,

$$
K_{h=0}(t)=\mathcal{N},
$$

which is typical for completely integrable systems [10]. At the two points, $h=0$ and $h=(\pi / 2)$, where the crossover time $t^{*}$ [Eq. (22)] formally diverges (and close to them, for finite $\ell$ ), our expansions in $t$ break down (see Appendix A and Fig. 4). The case $h=\pi / 2$ actually corresponds to a generic realization of a Floquet time crystal $[29,40]$, which is nonergodic and where the discrete translational invariance in time is spontaneously broken. This corresponds to a staggered behavior of the spectral form factor:

$$
K_{h=\pi / 2}(t)=\left\{\begin{array}{ll}
\mathcal{N} & t \text { even } \\
0 & t \text { odd }
\end{array} .\right.
$$

We note that in the range $h \in(\pi / 4, \pi / 2)$, the RPM spectral form factor Eq. (21) in the non-RMT regime, $t<t^{*}$, still displays characteristic period-2 oscillations.

Carefully subtracting double-counted terms where exactly one configuration (word) in the string ("orbit") $\underline{s}_{1}, \underline{s}_{2}, \ldots, \underline{s}_{t}$ appears twice, one obtains exactly the RMT result Eq. (7) up to the second order:

$$
K(t)=2 t-2 t^{2} / 2^{\ell}+\mathcal{O}\left(t^{3} / 4^{\ell}\right)
$$

(see Appendix B). This can be considered as a many-qubit analogy of the Sieber-Richter pairing mechanism $[11,12]$. We conjecture that it should be possible to obtain a RMT result to all orders by implementing the multiple-counting technique generalizing the diagrammatics sketched in Appendixes A and B.

\section{KICKED TRANSVERSE FIELD ISING MODEL: THEORY EXPLAINS NUMERICS}

We compare analytic results for the RPM to exact numerical computations of the spectral form factor in the following family of kicked Ising models:

$$
J_{x}^{1}=a+\frac{N_{1} b}{x^{\alpha}}, \quad J_{x, x^{\prime}}^{2}=\frac{N_{2} J}{\left(x^{\prime}-x\right)^{\alpha}}, \quad J_{x, x^{\prime} \ldots}^{k>2} \equiv 0,
$$

with normalization constants defined as

$$
\frac{1}{N_{1}}=\sum_{x} \frac{1}{x^{\alpha}}, \quad \frac{1}{N_{2}}=\frac{1}{\ell-1} \sum_{x<x^{\prime}} \frac{1}{\left(x^{\prime}-x\right)^{\alpha}},
$$

and interaction effectively being short range, $N_{1,2}=$ $\mathcal{O}\left(\ell^{0}\right)$ for $\alpha>1$. Power-law decaying one-spin terms and two-spin interactions are motivated by a requirement for the spectrum of $H_{0}$ to be nondegenerate and free from any other discrete symmetry, which should be a generic situation. Our results are not sensitive to the exact choice of $\alpha$, as long as we are sufficiently far away from either strictly 


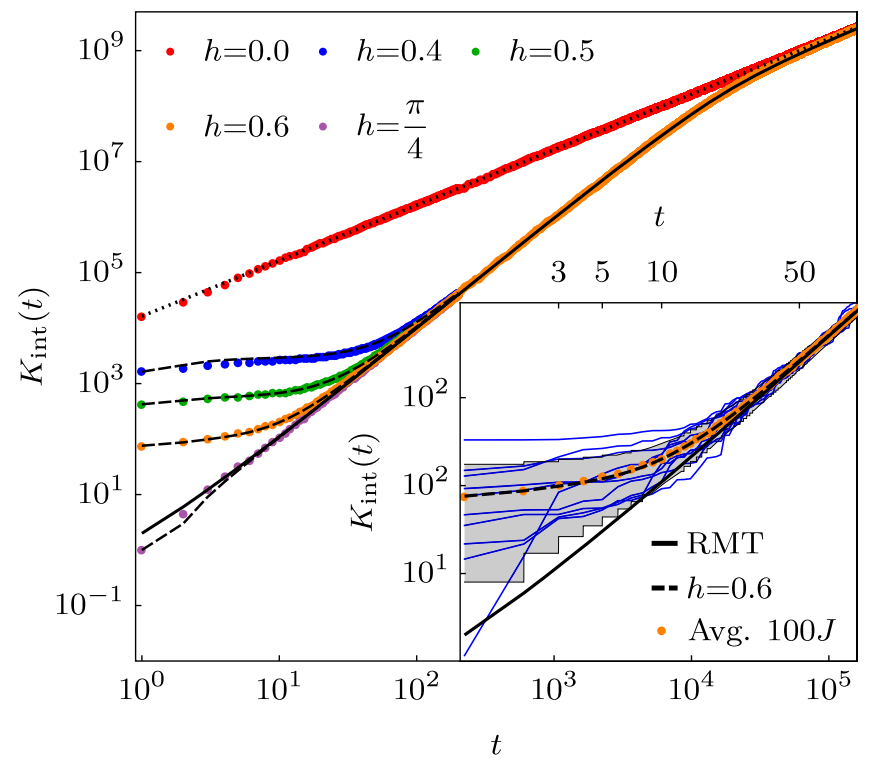

FIG. 1. Time-integrated spectral form factor for the kicked Ising model for up to ten Heisenberg times and at four different values of the transverse field $h$. The black dashed lines show predictions of the random phase model, while dotted (solid) lines give the Poissonian (RMT OE) result. The colored dots show numerical data averaged over 100 realizations of $J$ sampled uniformly in the interval $[5.5,55]$, for the kicked Ising model introduced in Eq. (26). In the inset we show that averaging is only necessary for very short times. Thin blue lines are particular realizations of the model for $J=10,11, \ldots 19$ and the shaded area shows the second to ninth decile assuming exponential distribution for realizations of $K(t)$ resulting in a hypoexponential distribution for the integrated spectral form factor. Other parameters are fixed to $\ell=14, a=1, b=5, \alpha=1.5$. local interactions $\alpha=\infty$ or very long-ranged interactions $\alpha \approx 0$, where the model becomes mean-field-like and describable by a single semiclassical degree of freedom.

In order to avoid the need of ensemble averaging, we define a time-integrated spectral form factor as

$$
K_{\text {int }}(t)=\sum_{\tau=1}^{t} K(\tau)
$$

shown in Fig. 1, which is indeed a self-averaging quantity as demonstrated in the inset. We observe very good agreement with the RPM. As $K_{\text {int }}(t)$ propagates deviations at short times to longer times, we also show the non-selfaveraging $K(t)$ at short times in Fig. 2 and again observe very good agreement with RPM upon averaging over an ensemble of values of parameter $J$ or taking a moving time average over a short window of time for fixed $J$ (and $a, b$, $\alpha$ ). Even the fluctuations of the two averages around the theoretical prediction (RPM) for similar statistical sample size $(n=10)$ look quantitatively comparable.

The data reported in Figs. 1 and 2 were obtained for locality exponent $\alpha=3 / 2$. In Fig. 3, we investigate the role of $\alpha$ in more detail. When the model becomes increasingly short ranged, i.e., increasing $\alpha$, the fluctuations part in Eq. (17) can no longer be neglected and the model develops deviations from RPM. The crossover time $t^{*}$ at which $K(t)$ begins to follow RMT becomes larger and starts to depend on the parameter $J$. But even for strictly local, nearestneighbor interactions $(\alpha \rightarrow \infty)$ at fixed $J$ this time seems to scale polynomially, perhaps like $\propto \ell^{2}$, and is still much smaller than the Heisenberg time $t_{H}=2^{\ell}$.

In order to quantify a transition between RPM and nonRPM physics, we define the following order parameter:

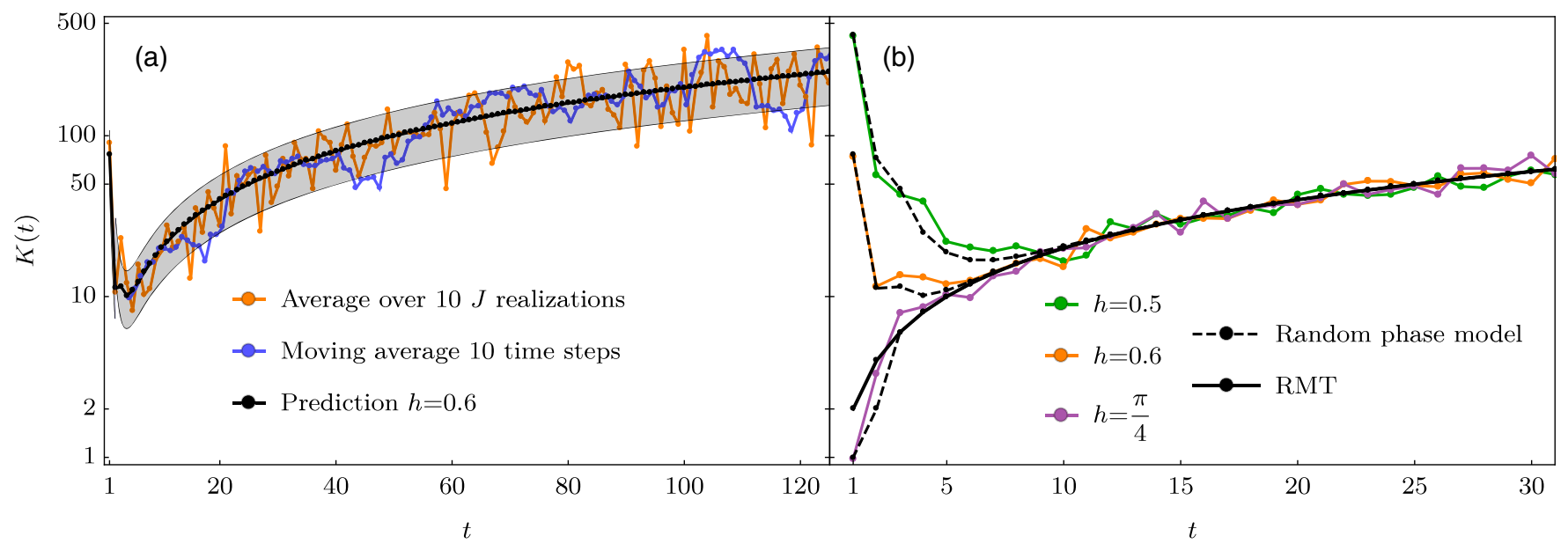

FIG. 2. Spectral form factor for the kicked Ising model at short times. (a) Comparison of a moving average over ten consecutive time steps and fixed model realization $(J=10)$ with the averaging over ten realizations $J=10,11, \ldots, 19$. All other parameters are fixed to $\ell=14, a=1, b=5, \alpha=1.5$. Shaded area shows the second to ninth decile assuming exponential distribution for realizations of $K(t)$ resulting in the gamma distribution for the averages. (b) $J$-averaged spectral form factor (now averaged over a sample of 100 values of $J \in[5.5,55])$ at short times shows deviations from the RMT, and the deviations are well captured by the random phase model. 

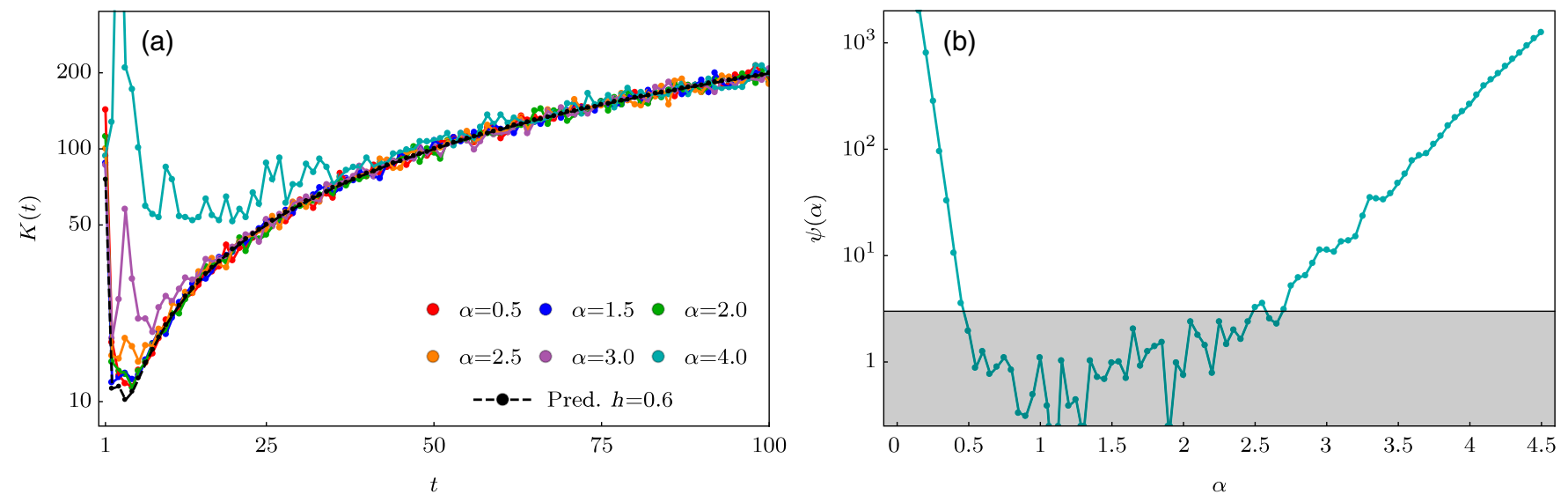

FIG. 3. (a) Spectral form factor for the kicked Ising model at short times for different values of locality exponent $\alpha$. When the model becomes increasingly short ranged, it develops deviations at short times different from the prediction of the random phase model. (b) The order parameter as defined in Eq. (29) for different values of $\alpha$, which shows that the model starts to develop deviations from the random phase model when $\alpha \lesssim 0.5$ or $\alpha \gtrsim 2.5$. The shaded area guides the eye to the section with small, statistically insignificant deviations. Averaging over 500 realizations of $J \in[5.5,255]$ is performed, other parameters are fixed to $\ell=14, a=1, b=5$, and $t_{\max }=100$.

$$
\psi(\alpha)=\left|\sum_{\tau=1}^{t_{\max }} \frac{K_{\mathrm{RPM}}(\tau)-K(\tau)}{K_{\mathrm{RPM}}(\tau)}\right| .
$$

Since the RPM prediction for long times becomes equivalent to RMT and is expected to match $K(t)$ well, provided the model is nonintegrable and ergodic, the order parameter $\psi$ becomes independent of $t_{\max }$ as long as $t^{*} \ll t_{\max } \ll t_{H}$. As shown in Fig. 3, we indeed find a phase-transitionlike behavior of $\psi(\alpha)$ with two, short-range and longrange critical points, $\alpha_{\text {long }}^{*} \sim 0.5$ and $\alpha_{\text {short }}^{*} \sim 2.5$ : For $\alpha_{\text {long }}^{*}<\alpha<\alpha_{\text {short }}^{*}$, agreement with RPM is excellent and $\psi(\alpha)$ is small and of the order of expected statistical fluctuation due to averaging over $J$, while outside the range, $\psi(\alpha)$ quickly grows.

\section{CONCLUSION}

Our work discloses the first theoretical mechanism which connects RMT to simple many-qubit systems in (effectively) low dimensions. There are many immediate further questions and generalizations which are to be studied. (i) The assumption of the pure $\delta$ correlator of phases Eq. (17) is on the same level of rigor as the random phase approximation in standard semiclassics, but one may hope to find a more rigorous justification here. (ii) The interesting case of local Ising interactions $(\alpha=\infty)$ also obeys RMT physics [38] but needs to be studied separately as the rhs of Eq. (17) then acquires extra systematic contributions. (iii) One may generalize our technique to study universal behavior of dynamical correlation functions (i.e., spin structure factors) in the quantum chaotic regime. (iv) Furthermore, one may expand our methods by introducing quenched disorder, say, in the transverse field, and attempt to approach the many-body localization transition [25] from the ergodic side, for instance, by tuning the locality exponent $\alpha$.

Our results have a direct relevance for understanding the vast body of numerical experiments, simulations, and in the near future possibly also experimental spectra of highly excited simple many-body systems, which correspond to ever longer accessible observation times of perfectly coherent out-of-equilibrium quantum systems (see, e.g., Ref. [30]). The ideas of many-body quantum chaos and random matrix theory are also vividly debated in the context of high-energy physics and holography [41-43], where our results and methods could also be applied.

Recently, we learned of a series of related works [44,45], where the RMT spectral form factor has been computed for a local Haar-random unitary nearest-neighbor quantum circuit propagator, which corresponds to the UE universality class of RMT, in the limit of large local Hilbert space dimension $q$. It is remarkable that in Ref. [45], where the authors consider a related variant of RPM, but insist on the strict locality of the (nearest-neighbor) interaction at the expense of having to consider a large $q$ limit, they found the exact same scaling of the Ehrenfest-Thouless timescale $t^{*} \propto \log \ell$.

\section{ACKNOWLEDGMENTS}

We thank B. Bertini, M. Horvat, and B. Žunkovič for discussions and T. H. Seligman for useful comments on the manuscript. The work is supported by Advanced grant of European Research Council (ERC) 694544-OMNES, as well as Grants No. P1-0044, N1-0025 and No. N1-0055 of Slovenian Research Agency. 
APPENDIX A: DIAGRAMMATIC EXPANSION OF THE LEADING CORRECTIONS

When computing $K(t)$, Eq. (18), we have to sum over all permutations $\pi \in S_{t}$. As we already noted, the (anti)cyclic permutations together with the identity permutation, which form a subgroup of $S_{t}$ (the so-called dihedral group), yield identical leading contributions which become exponentially (in $\ell$ ) dominant in the thermodynamic limit. Here, we identify and explicitly calculate the contributions of the permutations which yield the leading (first-order) corrections. From Eq. (20) it follows that each contribution to $Z_{\pi}$ depends only on the number of domain walls in periodic strings $\underline{s}=\left(s_{1}, s_{2}, \ldots, s_{t}\right)$ and $\pi(\underline{s})=\left(s_{\pi_{1}}, s_{\pi_{2}}, \ldots, s_{\pi_{t}}\right)$. Therefore, $Z_{\pi}$ depends solely on a diagram obtained by plotting a directed graph of sequentially arranged nodes $\left(s_{1}, s_{2}, \ldots, s_{t}\right)$ with the links $s_{\pi_{1}} \rightarrow s_{\pi_{2}}, s_{\pi_{2}} \rightarrow s_{\pi_{3}}, \ldots, s_{\pi_{t}} \rightarrow s_{\pi_{1}}$. For example, the (anti)cyclic permutations are then represented as circular loops, meaning that they preserve sequential order. The next-to-leading order $Z_{X}$ comes from the so-called $X$ diagrams (shown in the diagrammatic expression below and illustrated numerically in Fig. 4), where all connections apart from two (order changes) are kept intact (sequential). The diagrammatic expression for the case where the first sequential stretch has length $\tau$, and the second length $t-\tau-2$, reads

$$
Z_{\mathrm{X}}(\tau)=\sum_{s_{\tau}, s_{\tau+1}, s_{t-1}, s_{t}} T_{s_{t}, s_{\tau}}^{\tau} T_{s_{\tau+1}, s_{t-1}}^{t-\tau-2} v_{s_{\tau}, s_{\tau+1}} v_{s_{\tau}, s_{t-1}}^{*} v_{s_{t-1}, s_{t}} v_{s_{\tau+1}, s_{t}}^{*}=\frac{1}{2}\left(1+\lambda^{\tau}+\lambda^{t-\tau-2}-\lambda^{t-2}+\lambda^{t}\right)
$$

Circular black arcs represent summations over stretches of sequential spins $\left[\tau^{\prime}, \tau^{\prime \prime}\right]$, namely, over all $s_{\tau}, \tau^{\prime}<\tau<\tau^{\prime \prime}$. These are given by the powers of the transfer matrix $T_{\tau_{\tau^{\prime}}, s_{\tau^{\prime \prime}}^{\tau^{\prime \prime}}}^{\tau^{\prime}+1}$, specifically, $T_{s s^{\prime}}^{p}=\frac{1}{2}\left[1+(-1)^{s-s^{\prime}} \lambda^{p}\right]$, where $\lambda=\cos 2 h$ plays the role of the coupling constant. Red dots correspond to remaining spins, which one still needs to sum over while putting the matrix element $v_{s s^{\prime}}$ for each broken sequential link $s \rightarrow s^{\prime}$ (dotted) and $v_{s s^{\prime}}^{*}$ for each crossed link $s \rightarrow s^{\prime}$.

Of similar importance are the $X X$ diagrams with two sequential crossings:

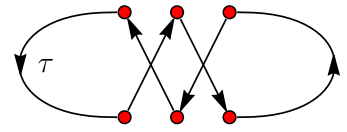

which are straightforwardly evaluated:

$$
Z_{X X}(\tau)=\frac{1}{2}\left[1+\lambda^{\tau+2}+\lambda^{t-\tau-2}+2 \lambda^{t-4}\left(\lambda^{4}-\lambda^{2}+\frac{1}{2}\right)\right] .
$$

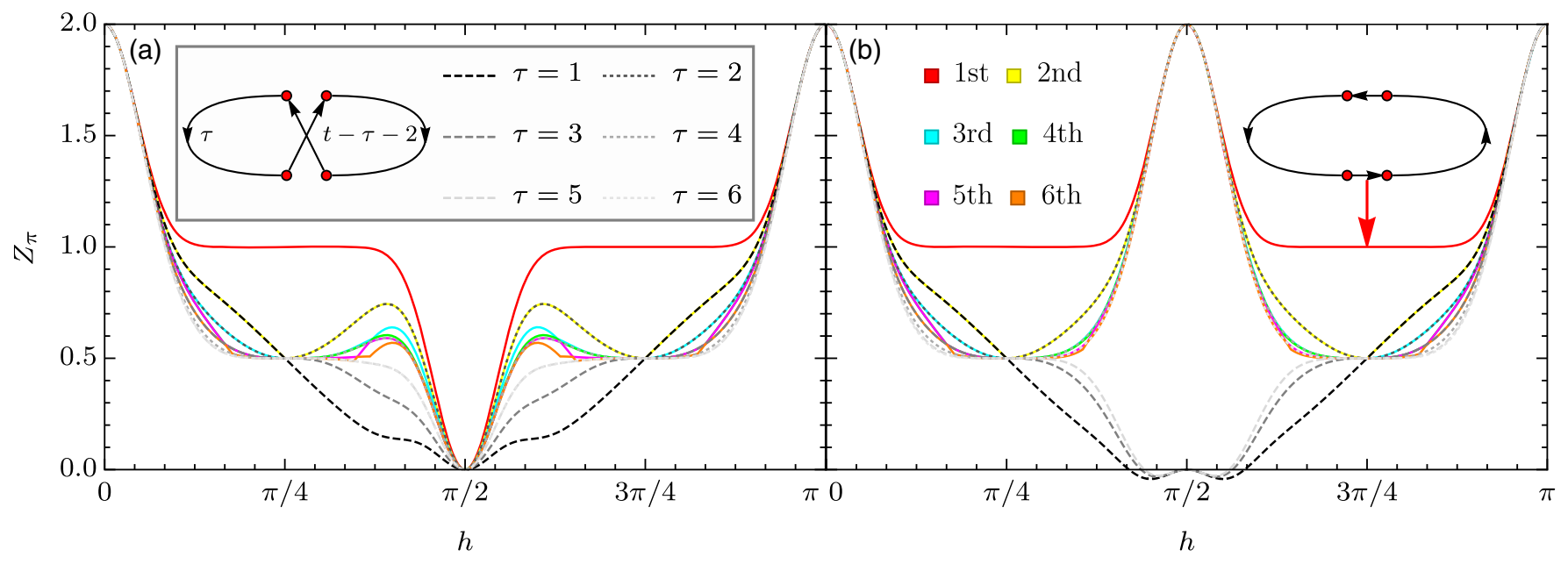

FIG. 4. Partition function $Z_{\pi}$ of the twisted one-dimensional Ising model for different permutation families is plotted against the field parameter $h$. Since in the expression the contribution occurs at a large power $\ell$, only the largest contribution matters in the first order (red), which corresponds to (anti)cyclic permutations. The first subleading corrections are given by the $X$ diagrams for $\tau=1,2$. The colored lines are numerical data for $t=13,14$ shown in (a) and (b), respectively. Dashed lines are the exact expressions for $Z_{X}(\tau)$ given by Eq. (A1). Note that all terms have multiplicities which are multiples of $2 t$. 
One can show that contributions of all other diagrams, starting with two crossings separated by sequential stretches, triple crossings, etc., are of the form $Z_{\text {other }}(\tau)=2^{-n}\left[1+\mathcal{O}\left(\lambda^{k}\right)\right]$, with $n \geq 2$ and $k \geq 1$, so they contribute to $Z^{\ell}$ only beyond the second order in $t / 2^{\ell}$ and are ignored here.

Each $X$ and $X X$ diagram (for fixed $\tau$ ) has multiplicity $t^{2}$ (or $t^{2} / 2$ for $\tau=t / 2$ ), since any $Z_{\pi}$ is invariant under $2 t$ cyclic and anticyclic permutations and we can start drawing the diagram at $t / 2$ inequivalent points.

We now see that the smallest gap of $Z_{\text {id }}-Z_{\pi}>0$ comes from $Z_{X}(1)$ for $\lambda>0$ and $Z_{X}(2)$ for $\lambda<0$ (see Fig. 4). Since these terms enter to power $\ell$, the subleading contributions to $K(t)$ are exponentially suppressed by a factor of the order of $\sim t[(1+\lambda) / 2]^{\ell}$ for $\lambda>0$ and $t\left[\left(1+\lambda^{2}\right) / 2\right]^{\ell}$ for $\lambda<0$. When we approach the Heisenberg time $t \sim t_{H}=2^{\ell}$, these contributions become important, as we see in Appendix B.

\section{APPENDIX B: SECOND-ORDER TERM IN $t / t_{H}$}

RMT predicts that the next term in the expansion is $-2 t^{2} / 2^{\ell}$, Eq. (7). We now show that the contributions of the $X$ and $X X$ diagrams and the possible repetitions of the spin configurations almost cancel, yielding exactly the RMT result.

In the first-order approximation in $t / 2^{\ell}$, we neglect the possibility that the spin configurations $\underline{s}_{\tau}, \tau=1, \ldots, t$, can repeat after some time. The leading-order correction to this consists of cases with a single repetition $\underline{s}_{\tau_{1}}=\underline{s}_{\tau_{2}}$. Then, Eq. (17) renders the permutation $\pi$ to run over a factor group $S_{t} / S_{\left\{\tau_{1}, \tau_{2}\right\}}$, where $S_{\left\{\tau_{1}, \tau_{2}\right\}}$ is a two-element permutation group. Because our leading-order sum Eq. (18) still runs over the entire permutation group $S_{t}$, we end up counting each element twice, so we need to subtract the overcounted terms,

$$
K(t)=\sum_{\pi \in S_{t}} Z_{\pi}^{\ell}-\sum_{1 \leq \tau_{1}<\tau_{2} \leq t} \frac{1}{2} \sum_{\pi \in S_{t}} \sum_{\underline{s}_{1}, \ldots, \underline{s}_{t}}^{\underline{s}_{\tau_{1}}=\underline{s}_{\tau_{2}}} F_{\pi}(\underline{\underline{s}})=\sum_{\pi \in S_{t}} Z_{\pi}^{\ell}-\frac{1}{2} \frac{t}{2} \sum_{\tau=1}^{t-1} \sum_{\pi \in S_{t}} \sum_{\underline{\underline{s}}}^{\underline{s}_{\tau}=\underline{s_{t}}} F_{\pi}(\underline{\underline{s}}),
$$

where $F_{\pi}(\underline{\underline{s}})=\prod_{x=1}^{\ell} \prod_{\tau=1}^{t} v_{s_{x, \tau}, s_{x, \tau+1}} v_{s_{x, \tau}^{\prime}, s_{x, \tau+1}^{\prime}}^{*}$ and $\underline{\underline{s}} \equiv\left(s_{x, \tau} ; 1 \leq x \leq \ell, 1 \leq \tau \leq t\right)$. In the second line we use time invariance to set $\tau_{2}=t$ and $\tau_{1}=\tau$. Since the repeated spin contributes the same regardless of its value, the sum trivializes for $\pi=\mathrm{id}$ to a product of sums for separate spins, each contributing

$$
\sum_{\underline{s}}^{s_{\tau}=s_{t}} F_{\pi}^{\ell=1}(\underline{s})=2 T_{00}^{\tau} T_{00}^{t-\tau}=\frac{1}{2}\left(1+\lambda^{\tau}+\lambda^{t-\tau}+\lambda^{t}\right) .
$$

Following the same argument as before, invariance under cyclic and anticyclic permutations again yields multiplicity $2 t$. But because of the repetition of spin configurations, the $X$ and $X X$ diagrams where the crossed link contains the repeated spin yield the contribution in the same order $\frac{1}{2}\left[1+\mathcal{O}\left(\lambda^{\tau}\right)\right]$ :

$$
\begin{aligned}
K(t) & =2 t\left(1+\lambda^{t}\right)^{\ell}+\frac{t^{2}}{2^{\ell}}\left(\sum_{\tau=1}^{t-3}\left(1+\lambda^{\tau}+\lambda^{t-\tau-2}-\lambda^{t-2}+\lambda^{t}\right)^{\ell}\right. \\
& +\sum_{\tau=3}^{t-3}\left(1+\lambda^{\tau}+\lambda^{t-\tau}+\lambda^{t-4}\left(2 \lambda^{4}-2 \lambda^{2}+1\right)\right)^{\ell} \\
& -\sum_{\tau=1}^{t-1}\left(1+\lambda^{\tau}+\lambda^{t-\tau}+\lambda^{t}\right)^{\ell} \\
& \left.-\sum_{\tau=3}^{t-3}\left(1+\lambda^{\tau}+\lambda^{t-\tau}+\lambda^{t}\right)^{\ell}\right)+\ldots \\
& =2 t-\frac{2 t^{2}}{2^{\ell}}+\mathcal{O}\left(\frac{t^{3}}{4^{\ell}}\right) .
\end{aligned}
$$

On the right-hand side, the corresponding diagrams are shown for clarity, where the blue diamond sites connected with a dashed line depict the repeating spin. The first two sums come from enumerating all $X$ and $X X$ diagrams (as explained in Appendix A). The sum from the repeating spin configurations comes next, and is written in two parts. The combinatorial factor of the diagrams is $4 t$ when $\tau=1,2$, $t-1, t-2$ and $8 t$ otherwise, which we take into account by 
writing two sums with different starting and final values of $\tau$. In the last line of Eq. (B3) we note a remarkable cancellation of all terms apart from the RMT result for times $t>t^{\diamond}=\mathcal{O}(\ell \log \lambda)$, where $t_{H} / t^{\diamond}$ is still exponentially large in $\ell$. This could be viewed as a quantum many-body analogy of the Sieber-Richter self-encountering-orbits mechanism.

[1] E. P. Wigner, Characteristic Vectors of Bordered Matrices with Infinite Dimensions, Ann. Math. 62, 548 (1955).

[2] R. U. Haq, A. Pandey, and O. Bohigas, Fluctuation Properties of Nuclear Energy Levels: Do Theory and Experiment Agree?, Phys. Rev. Lett. 48, 1086 (1982).

[3] N. Rosenzweig and C. E. Porter, "Repulsion of Energy Levels" in Complex Atomic Spectra, Phys. Rev. 120, 1698 (1960).

[4] S. W. McDonald and A. N. Kaufman, Spectrum and Eigenfunctions for a Hamiltonian with Stochastic Trajectories, Phys. Rev. Lett. 42, 1189 (1979).

[5] G. Casati, F. Valz-Gris, and I. Guarneri, On the Connection between Quantization of Nonintegrable Systems and Statistical Theory of Spectra, Lett. Nuovo Cimento Soc. Ital. Fis. 28, 279 (1980).

[6] M. V. Berry, Quantizing a Classically Ergodic System: Sinai's Billiard and the KKR Method, Ann. Phys. (N.Y.) 131, 163 (1981).

[7] O. Bohigas, M. J. Giannoni, and C. Schmit, Characterization of Chaotic Quantum Spectra and Universality of Level Fluctuation Laws, Phys. Rev. Lett. 52, 1 (1984).

[8] M. V. Berry, Semiclassical Theory of Spectral Rigidity, Proc. R. Soc. A 400, 229 (1985).

[9] F. Haake, Quantum Signatures of Chaos, 2nd ed. (Springer, New York, 2001).

[10] M. V. Berry and M. Tabor, Level Clustering in the Regular Spectrum, Proc. R. Soc. A 356, 375 (1977).

[11] M. Sieber and K. Richter, Correlations between Periodic Orbits and Their Role in Spectral Statistics, Phys. Scr. T T90, 128 (2001).

[12] M. Sieber, Leading Off-Diagonal Approximation for the Spectral Form Factor for Uniformly Hyperbolic Systems, J. Phys. A 35, L613 (2002).

[13] S. Müller, S. Heusler, P. Braun, F. Haake, and A. Altland, Semiclassical Foundation of Universality in Quantum Chaos, Phys. Rev. Lett. 93, 014103 (2004).

[14] S. Müller, S. Heusler, P. Braun, F. Haake, and A. Altland, Periodic-Orbit Theory of Universality in Quantum Chaos, Phys. Rev. E 72, 046207 (2005).

[15] Z. Pluhar and H. A. Weidenmüller, Universal Quantum Graphs, Phys. Rev. Lett. 112, 144102 (2014).

[16] Z. Pluhar and H. A. Weidenmüller, Quantum Graphs and Random-Matrix Theory, J. Phys. A 48, 275102 (2015).

[17] T. Engl, J. Dujardin, A. Argüelles, P. Schlagheck, K. Richter, and J.-D.Urbina, Coherent Backscattering in Fock Space: A Signature of Quantum Many-Body Interference in Interacting Bosonic Systems, Phys. Rev. Lett. 112, 140403 (2014).
[18] T. Engl, J.-D. Urbina, and K. Richter, Periodic Mean-Field Solutions and the Spectra of Discrete Bosonic Fields: Trace Formula for Bose-Hubbard Models, Phys. Rev. E 92, 062907 (2015).

[19] M. Akila, D. Waltner, B. Gutkin, P. Braun, and T. Guhr, Semiclassical Identification of Periodic Orbits in a Quantum Many-Body System, Phys. Rev. Lett. 118, 164101 (2017).

[20] R. Dubertrand and S. Müller, Spectral Statistics of Chaotic Many-Body Systems, New J. Phys. 18, 033009 (2016).

[21] G. Montambaux, D. Poilblanc, J. Bellissard, and C. Sire, Quantum Chaos in Spin-Fermion Models, Phys. Rev. Lett. 70, 497 (1993).

[22] T. Prosen, Ergodic Properties of a Generic Nonintegrable Quantum Many-Body System in the Thermodynamic Limit, Phys. Rev. E 60, 3949 (1999).

[23] L. F. Santos and M. Rigol, Localization and the Effects of Symmetries in the Thermalization Properties of OneDimensional Quantum Systems, Phys. Rev. E 82, 031130 (2010).

[24] C. Kollath, G. Roux, G. Biroli, and A. Laeuchli, Statistical Properties of the Spectrum of the Extended Bose-Hubbard Model, J. Stat. Mech. (2010) P08011.

[25] R. Nandkishore and D. A. Huse, Many-Body Localization and Thermalization in Quantum Statistical Mechanics, Annu. Rev. Condens. Matter Phys. 6, 15 (2015).

[26] M. Serbyn and J. E. Moore, Spectral Statistics Across the Many-Body Localization Transition, Phys. Rev. B 93, 041424(R) (2016).

[27] D. J. Luitz, N. Laflorencie, and F. Alet, Many-Body Localization Edge in the Random-Field Heisenberg Chain, Phys. Rev. B 91, 081103(R) (2015).

[28] P. Ponte, Z. Papic, F. Huveneers, and D. A. Abanin, ManyBody Localization in Periodically Driven Systems, Phys. Rev. Lett. 114, 140401 (2015).

[29] V. Khemani, A. Lazarides, R. Moessner, and S. L. Sondhi, Phase Structure of Driven Quantum Systems, Phys. Rev. Lett. 116, 250401 (2016).

[30] P. Bordia, H. Lüschen, U. Schneider, M. Knap, and I. Bloch, Periodically Driving a Many-Body Localized Quantum System, Nat. Phys. 13, 460 (2017).

[31] M. Rigol, D. Vanja, and O. Maxim, Thermalization and Its Mechanism for Generic Isolated Quantum Systems, Nature (London) 452, 854 (2008).

[32] M. L. Mehta, Random Matrices and the Statistical Theory of Spectra, 2nd ed. (Academic, New York, 1991).

[33] R. E. Prange, The Spectral Form Factor Is Not SelfAveraging, Phys. Rev. Lett. 78, 2280 (1997).

[34] J. H. Hannay and A. M. Ozorio De Almeida, Periodic Orbits and a Correlation Function for the Semiclassical Density of States, J. Phys. A 17, 3429 (1984).

[35] K. Saito, T. Nagao, S. Müller, and P. Braun, Semiclassical Theory for Universality in Quantum Chaos with Symmetry Crossover, J. Phys. A 42, 495101 (2009).

[36] T. Prosen, General Relation Between Quantum Ergodicity and Fidelity of Quantum Dynamics, Phys. Rev. E, 65, 036208 (2002). 
[37] T. Prosen, Chaos and Complexity of Quantum Motion, J. Phys. A 40, 7881 (2007).

[38] C. Pineda and T. Prosen, Universal and Nonuniversal Level Statistics in a Chaotic Quantum Spin Chain, Phys. Rev. E 76, 061127 (2007).

[39] M. Akila, D. Waltner, B. Gutkin, and T. Guhr, Particle-Time Duality in the Kicked Ising Spin Chain, J. Phys. A 49, 375101 (2016).

[40] D. V. Else, B. Bauer, and C. Nayak, Floquet Time Crystals, Phys. Rev. Lett. 117, 090402 (2016).

[41] D. A. Roberts, D. Stanford, and L. Susskind, Localized Shocks, J. High Energy Phys. 03 (2015) 51.
[42] J. Maldacena, S. H. Shenker, and D. Stanford, A Bound on Chaos, J. High Energy Phys. 08 (2016) 106.

[43] J. S. Cotler, G. Gur-Ari, M. Hanada, J. Polchinski, P. Saad, S. H. Shenker, D. Stanford, A. Streicher, and M. Tezuka, Black Holes and Random Matrices, J. High Energy Phys. 05 (2017) 118.

[44] A. Chan, A. De Luca, and J. T. Chalker, Solution of a Minimal Model for Many-Body Quantum Chaos, arXiv: 1712.06836.

[45] A. Chan, A. De Luca, and J. T. Chalker, Spectral Statistics in Spatially Extended Chaotic Quantum Many-Body Systems, arXiv:1803.03841. 